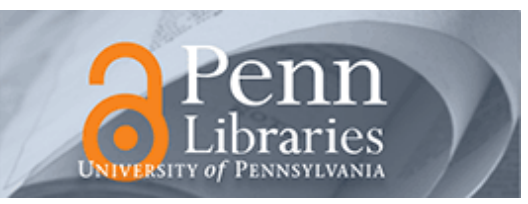

University of Pennsylvania

ScholarlyCommons

6-1-2009

\title{
Demonstration of Inverse Acoustic Band Gap Structures in AIN and Integration with Piezoelectric Contour Mode Transducers
}

\author{
Nai-Kuei Kuo \\ University of Pennsylvania, kuo1@seas.upenn.edu \\ Chengjie Zuo \\ University of Pennsylvania, chengjiezuo@hotmail.com \\ Gianluca Piazza \\ University of Pennsylvania, piazza@seas.upenn.edu
}

Follow this and additional works at: https://repository.upenn.edu/ese_papers

Part of the Electrical and Electronics Commons, and the Electro-Mechanical Systems Commons

\section{Recommended Citation}

Nai-Kuei Kuo, Chengjie Zuo, and Gianluca Piazza, "Demonstration of Inverse Acoustic Band Gap Structures in AIN and Integration with Piezoelectric Contour Mode Transducers", The 15th International Conference on Solid-State Sensors, Actuators and Microsystems (Transducers 2009), 2334-2337. June 2009.

Suggested Citation:

Nai-Kuei Kuo, Chengjie Zuo, and Gianluca Piazza. "Demonstration of Inverse Acoustic Band Gap Structures in AIN and Integration with Piezoelectric Contour Mode Transducers" The 15th International Conference on Solid-State Sensors, Actuators and Microsystems (Transducers 2009) (2009): 2334-2337.

(C)2009 IEEE. Personal use of this material is permitted. However, permission to reprint/republish this material for advertising or promotional purposes or for creating new collective works for resale or redistribution to servers or lists, or to reuse any copyrighted component of this work in other works must be obtained from the IEEE.

This paper is posted at ScholarlyCommons. https://repository.upenn.edu/ese_papers/539

For more information, please contact repository@pobox.upenn.edu. 


\title{
Demonstration of Inverse Acoustic Band Gap Structures in AIN and Integration with Piezoelectric Contour Mode Transducers
}

\begin{abstract}
This paper presents the first design and demonstration of a novel inverse acoustic band gap (IABG) structure in aluminum nitride (AIN) and its direct integration with piezoelectric contour-mode transducers. The experimental results indicate that the IABG structure has a stop band from $185 \mathrm{MHz}$ to $240 \mathrm{MHz}$ and is centered around $219 \mathrm{MHz}$ with maximum rejection of $30 \mathrm{~dB}$. The ABG-induced phonon scattering causes a frequency band gap that prohibits the propagation of certain acoustic wavelengths. In this work, the IABG unit cell consists of a high acoustic velocity $(V)$ center material, which is formed by 2 - $\mu m$-thick AIN sandwiched by $200-\mathrm{nm}$-thick platinum (Pt) and is held by four thin tethers and surrounded by a low acoustic velocity material (air). This cell arrangement enlarges the frequency band gap and eases the requirements on the thickness (d) to lattice constant (a) ratio, which was imposed by previous ABG demonstration in the very high frequency range. The finite element method (FEM) analysis indicates that the IABG can produce a gap-to-midgap ratio of $13.5 \%$ even when the $\mathrm{d} / \mathrm{a}$ ratio is as small as 0.23 . This advantage further allows the direct integration of the IABG with high frequency bulk acoustic wave (BAW) transducers.

\section{Disciplines}

Electrical and Electronics | Electro-Mechanical Systems

\section{Comments}

Suggested Citation:

Nai-Kuei Kuo, Chengjie Zuo, and Gianluca Piazza. "Demonstration of Inverse Acoustic Band Gap Structures in AIN and Integration with Piezoelectric Contour Mode Transducers" The 15th International Conference on Solid-State Sensors, Actuators and Microsystems (Transducers 2009) (2009): 2334-2337.

(C2009 IEEE. Personal use of this material is permitted. However, permission to reprint/republish this material for advertising or promotional purposes or for creating new collective works for resale or redistribution to servers or lists, or to reuse any copyrighted component of this work in other works must be obtained from the IEEE.
\end{abstract}




\title{
DEMONSTRATION OF INVERSE ACOUSTIC BAND GAP STRUCTURES IN AIN AND INTEGRATION WITH PIEZOELECTRIC CONTOUR MODE TRANSDUCERS
}

\author{
N. Kuo*, C. Zuo, and G. Piazza \\ University of Pennsylvania, Philadelphia, Pennsylvania, USA
}

\begin{abstract}
This paper presents the first design and demonstration of a novel inverse acoustic band gap (IABG) structure in aluminum nitride (AIN) and its direct integration with piezoelectric contour-mode transducers. The experimental results indicate that the IABG structure has a stop band from $185 \mathrm{MHz}$ to $240 \mathrm{MHz}$ and is centered around 219 $\mathrm{MHz}$ with maximum rejection of $30 \mathrm{~dB}$. The ABGinduced phonon scattering causes a frequency band gap that prohibits the propagation of certain acoustic wavelengths. In this work, the IABG unit cell consists of a high acoustic velocity $(V)$ center material, which is formed by $2-\mu \mathrm{m}$-thick AlN sandwiched by 200 -nm-thick platinum $(\mathrm{Pt})$ and is held by four thin tethers and surrounded by a low acoustic velocity material (air). This cell arrangement enlarges the frequency band gap and eases the requirements on the thickness $(d)$ to lattice constant $(a)$ ratio, which was imposed by previous $\mathrm{ABG}$ demonstration in the very high frequency range. The finite element method (FEM) analysis indicates that the IABG can produce a gap-to-midgap ratio of $13.5 \%$ even when the $d / a$ ratio is as small as 0.23 . This advantage further allows the direct integration of the IABG with high frequency bulk acoustic wave (BAW) transducers.
\end{abstract}

\section{KEYWORDS}

Acoustic band gap, phononic crystals, very high frequency (VHF) resonator, electro-acoustic transducer, microelectromechanical systems

\section{INTRODUCTION}

In recent years, research activities in phononic crystals (PCs) have been continuously increasing [1] because of the significant impact that PC-based devices can have on ultrasonic devices [2], RF communications, and heat transfer. However, there are only very few demonstrations of bulk acoustic band gap (ABG) in the Very High Frequency (VHF) range $[3,4]$. These ABG structures are based on a conventional cell arrangement having a high acoustic velocity $(V)$ matrix with center cylindrical low acoustic velocity inclusions. Large band gaps have been attained, but the bandwidths are highly dependent on the thickness, $d$, to lattice constant, $a$, ratio $(d / a)$. In Mohammadi's work [5], the optimum $d / a$ ratio is approximately 0.5 . This results in restrictions on the direct integration of $\mathrm{ABG}$ structures with high frequency $\mathrm{RF}$ MEMS devices, which generally require film thicknesses in the order of 1-2 $\mu \mathrm{m}$. Unlike these previous demonstrations, this work introduces a new class of $\mathrm{ABG}$ structures based on the use of a high acoustic velocity center media (AlN and $\mathrm{Pt}$ ) held by four thin tethers in a low acoustic velocity matrix (air). According to the resonance-induced acoustic band gap theory [3], the bandwidth of the IABG (inclusion acoustic velocity >> matrix acoustic velocity) is dominated by overlapping Bragg resonances. In other words, the bandwidth of the gap depends on the mismatch between the acoustic impedances and separation between the two Bragg fundamental frequencies. The IABG structure not only satisfies the ultimate mismatch in acoustic impedance but also widens the separation between the Bragg fundamental resonances. Due to the large frequency band gap induced by this cell arrangement, the bandwidth dependence on the film thickness is lowered. Furthermore, metal layers can be deposited on the IABG structure without significantly affecting the band gap. This permits the direct integration of the IABG with BAW RF MEMS devices and improves the acoustic matching between the electro-acoustic transducer and the PC-based devices.

The direct integration of the PC with the AlN contourmode transducers requires the use of a film thickness well below the optimum value of the previously demonstrated VHF ABG designs. Instead, the IABG induces a large complete frequency stop band in the very high frequency range with a small $d / a$ ratio of 0.23 , even though top and bottom Pt layers are introduced into the basic cell. These metals can function as electrodes to route electrical signals to resonant RF MEMS devices suspended via IABG structures or can result in a better acoustic matching between the transducers and the PC.

\section{DESIGN OF INVERSE ACOUSTIC BAND GAP WITH CONTOUR-MODE TRANSDUCERS}

\section{The Inverse Acoustic Band Gap (IABG) Structure}

The unit cell of the IABG structure is presented in Figure 1 . The principal dimensions are represented by the lattice constant, $a$, the radius, $r$, and the tether width, $w$.

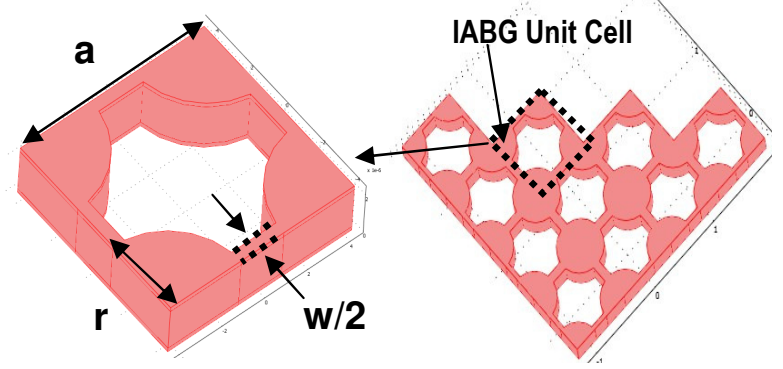

Figure 1: IABG unit cell and array. 
In this implementation, in order to directly integrate the IABG structure with contour-mode devices, the unit cell is formed by a $2-\mu \mathrm{m}$-thick AlN layer and $200-\mathrm{nm}$ thick top and bottom Pt layers. The predicted acoustic band gap induced by the structure is simulated by COMSOL $^{\circledR}$ Multi-physics software. This approach has been previously utilized for the calculation of complete acoustic band gaps [6]. The equation for an elastic wave traveling in a media with periodic acoustic properties can be expressed by the Bloch-Floquet theorem [7]. According to this theorem, the eigenfrequencies of each propagation mode can be found by applying periodic boundary conditions on the unit cell in $\mathrm{COMSOL}^{\circledR}$. The complete acoustic band gap is obtained by sweeping the eigenfrequency analysis over specific directions of the Brillouin zone ( $\Gamma-\mathrm{X}-\mathrm{M}-\Gamma)$ [8].

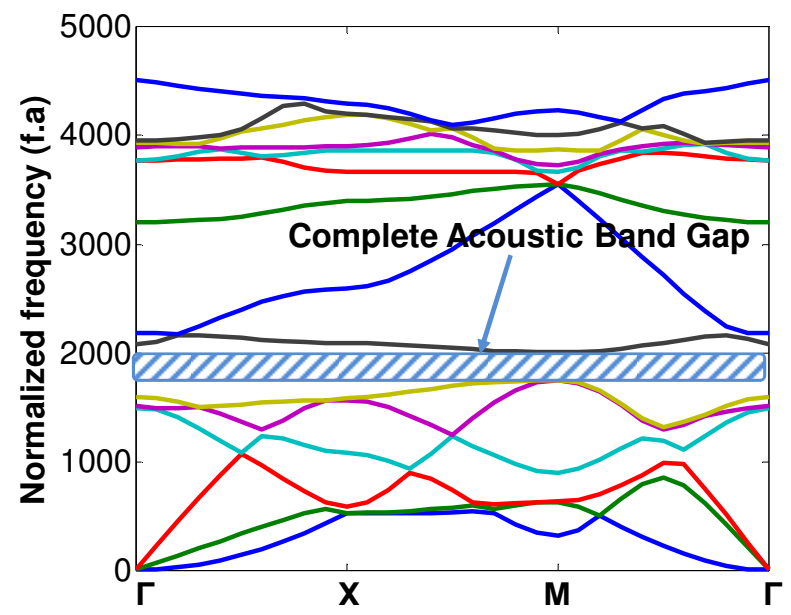

Figure 2: The dispersion relation between the wavevector and normalized frequency $(\mathrm{m} / \mathrm{s})$ obtained from COMSOL $®$ for the AlN unit cell shown in Fig. 1.

The normalized dispersion relation shown in Figure 2 was obtained for a unit cell with thickness of $2 \mu \mathrm{m}, r / a$ ratio of 0.38 , and tether width to lattice constant $(w / a)$ of 0.11 . These parameters were chosen in order to obtain the widest frequency band gap and simultaneously accommodate the fabrication tolerances (minimum feature size of $1 \mu \mathrm{m})$ and transducer thickness $(2 \mu \mathrm{m})$. In this first demonstration, VHF operation around $219 \mathrm{MHz}$ was selected. This frequency corresponds to an IABG having a lattice constant of $8.6 \mu \mathrm{m}$, radius of $3.3 \mu \mathrm{m}$, and tether width of $1 \mu \mathrm{m}$. This design corresponds to a complete band gap having a $13.5 \%$ gap-to-midgap ratio.

In this design, the tether width, $w$, plays an important role in defining the key characteristics of the acoustic band gap. The relationships between the width and the stop band simulated by $\mathrm{COMSOL}^{\circledR}$ are shown in Figure 3.

The FEM analysis indicates that the tether width significantly affects the IABG bandwidth whereas it has a limited impact on the center frequency. The large sensitivity to the tether width can be advantageously employed to further tune the gap-to-midgap ratio while maintaining the other cell dimensions unaltered. At the same time, this phenomenon negatively impacts the fabrication tolerances and renders the extension of this design to higher frequencies more challenging.
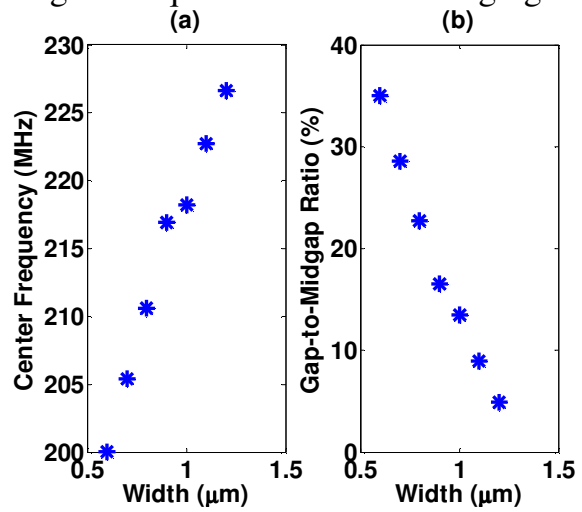

Figure 3: The relationship between acoustic band gap (a) center frequency and (b) bandwidth with respect to the tether width $(w)$. The other cell dimensions remain fixed: $a=8.6 \mu \mathrm{m}$, $r=3.3 \mu \mathrm{m}$

\section{AIN Piezoelectric Contour-Mode Transducers}

The AlN piezoelectric contour-mode (CM) transducers consist of two contour-mode resonators (CMRs), of which one serves as the transmitter of the acoustic signal, and the other as the receiver. This class of AlN resonators has already been investigated by the same group [9]. These electro-acoustic transducers function as couplers of electrical signals into acoustic waves, which are employed to test the IABG structure. Lateral bulk vibrations are in fact induced by the piezoelectric property of the AlN films, across which an electric field is applied via top and bottom Pt electrodes (Figure 4). Different from the previous demonstrations of ABGs [3, 4], the contour-mode transducers can produce in-plane acoustic waves directly coupled with and in the same plane of the IABG structure. The center frequency of the transducer depends on the width, $W$, of the individual fingers that form the resonant structure [9] and can be approximately predicted by analytical calculations.

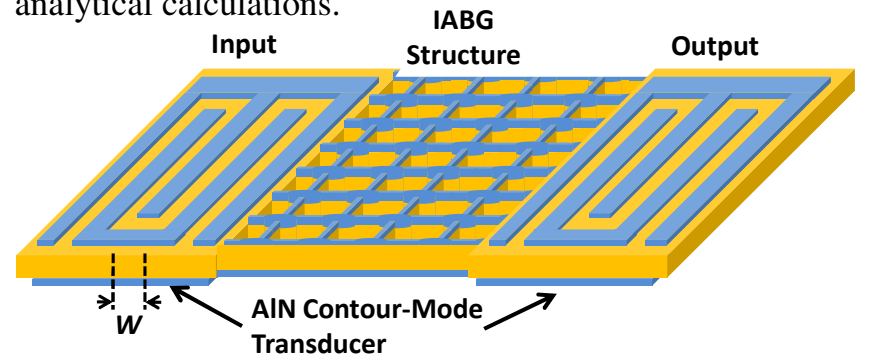

Figure 4: The realization of the AlN piezoelectric contourmode resonators coupled by IABG structures.

The bandwidth of these transducers is currently limited by the electromechanical coupling coefficient, $k_{t}^{2}$, of AlN and can be effectively used to cover at most $4 \%$ of the 
device center frequency [10]. This is not sufficient for testing the entire stop band of the IABG. For this reason, eight contour-mode transducers were designed to demonstrate the operation of the complete band gap. The same set of eight reference transducers coupling an AlN plate with top and bottom platinum, instead of the IABG array, was designed to normalize the transmission curves obtained in the experimental tests.

\section{FABRICATION PROCESS}

The fabrication process enables the direct integration of IABGs with contour-mode transducers. A completely top-down method already developed for CMRs [9] and based on a four-mask and post-CMOS compatible process was employed for the making of the IABGs. The four masks permit the definition of the bottom Pt electrodes, the via for the top and bottom electrodes connection, the top Pt electrodes, and the final AlN etching (Figure 5). The device was fabricated on top of a high resistivity (HR) silicon substrate (resistivity $>10^{4} \mathrm{ohm}-\mathrm{cm}$ ).

Figure 6 shows the scanning electron micrographs (SEMs) of the microfabricated IABGs integrated with AlN contour-mode transducers. The zoomed in view of the IABG unit cell shows some residual roughness induced by the AlN etch process. Although the experimental and theoretical data match quite closely, non-idealities in the microfabricated structures can affect the IABG response and will need to be taken into account into future and higher frequency implementations.

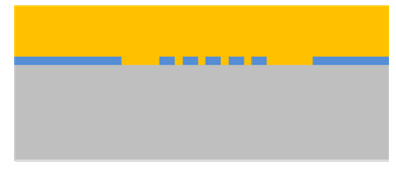

(a)

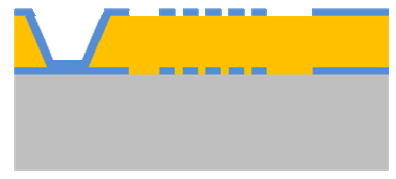

(c)

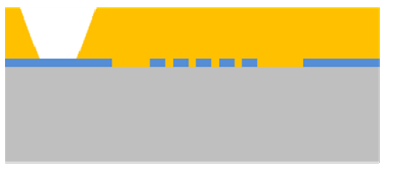

(b)

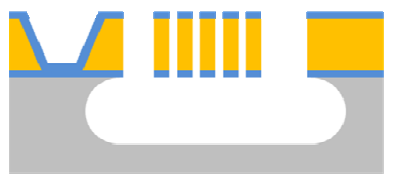

(d)

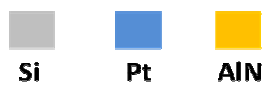

Figure 5: Fabrication process: Step (a), 2 m AlN layer was sputtered on top of the bottom Pt, which was sputtered and lifted off directly on the Si substrate. A thin Ti adhesion layer was employed. Step (b), by using $\mathrm{SiO}_{2}$ as protection mask for the AlN, a via was etched by AZ400K solution. The via is required in order to access the bottom electrode used to drive the high frequency AlN transducers. Step (c), the top Pt layers was deposited followed by lift off in an ultrasonic bath. Step (d), Inductively Coupled Plasma process was used to etch the AlN layer. Lastly, the device was released from the Si substrate by etching in $\mathrm{XeF}_{2}$.

\section{EXPERIMENTAL DISCUSSION \\ RESULTS}

AND

The transmission response of the IABG structures was measured via an Agilent N5230 PNA-L Network Analyzer after performing a standard short-open-load-through (SOLT) calibration. Each extracted $\mathrm{S}_{21}$ data is first impedance-matched for more effective normalization and to reduce reflection-induced losses in the acoustic structure. To demonstrate the operation of the full stop band, $4-5 \%$ bandwidth of the center frequency of each transducer transmission response was taken and summed to cover the entire frequency range of interest. After the summation over the various frequencies, the total transmission with IABG structures was normalized by the reference transmission of the plates without IABGs. In order to emphasize the stop band, the moving average method was used to trace the normalized transmission.

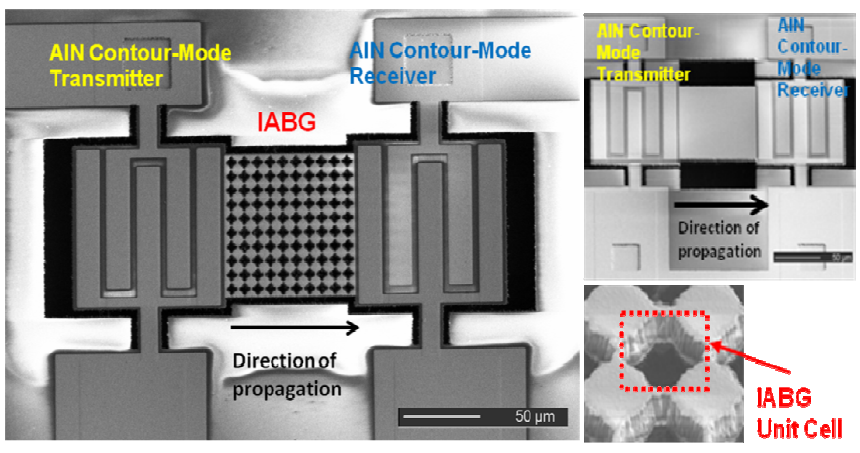

Figure 6: SEM image of the IABG array integrated with one of the piezoelectric MEMS transducers used to launch and sense acoustic waves through the IABG and a zoomed-in view of the $I A B G$ array, highlighting the unit cell and tiny connecting tethers. The inset to the right shows the image of a reference structure used to normalize the IABG response.

The experimental transmission of the IABG structures is shown in Figure 7. The effective frequency range determined by the $\mathrm{CM}$ transducers is from $175 \mathrm{MHz}$ to $260 \mathrm{MHz}$.

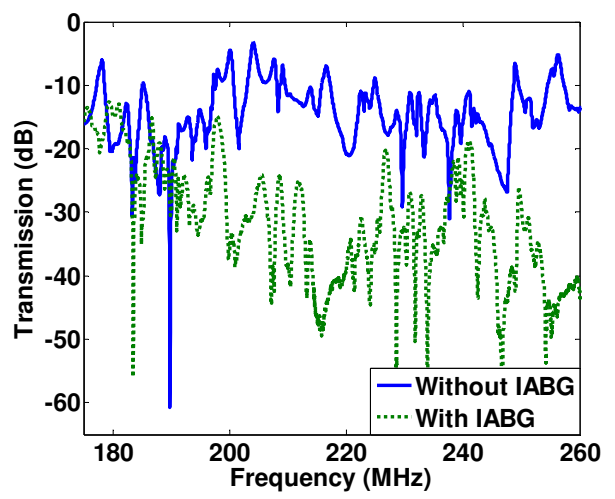

Figure 7: Comparison of the experimental transmission response for the devices with and without the IABG. Note the significant attenuation within the designed stop band between 190 and $240 \mathrm{MHz}$. 
The transmission response of Figure 7 indicates the presence of a larger stop band (>13.5\%) with respect to the simulated design value. This is a consequence of the fact that the CM devices induce acoustic waves primarily in one specific direction of propagation. In fact, the piezoelectric CM transducer generates waves mostly in the [100] direction $(\Gamma$ to $\mathrm{X})$. Figure 8 shows the simulated dispersion in the [100] direction compared with the experimental normalized transmission in the same frequency range.

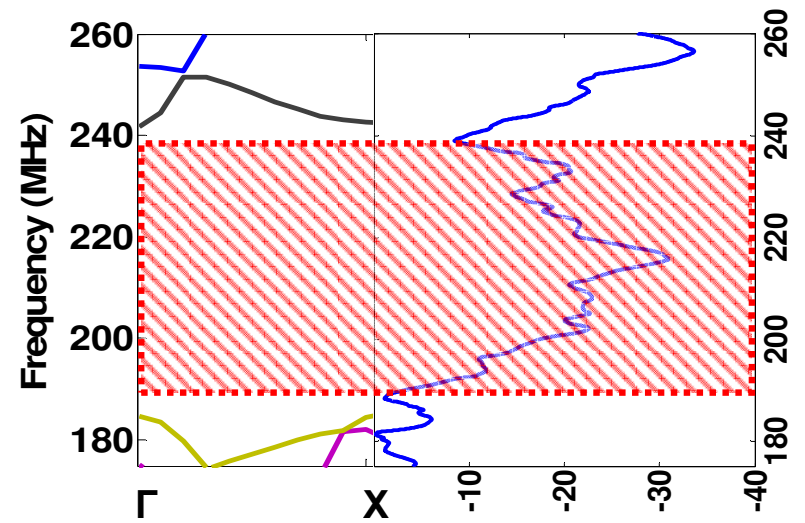

Figure 8: Comparison between the simulation and experimental results.

The COMSOL ${ }^{\circledR}$ analysis of Figure 8 confirms that a broader band gap exists in the $\Gamma$-X direction between 185 $\mathrm{MHz}$ and $240 \mathrm{MHz}$ as obtained experimentally.

The stop band shows a maximum rejection of $30 \mathrm{~dB}$. Limited rejection inside the band gap is likely due to imperfections in the fabrication process. Fabrication defects and sloped etching of the AlN films can in fact reduce the IABG rejection for certain wavelengths.

\section{CONCLUSION}

The first inverse acoustic band gap (IABG) structure in aluminum nitride was designed and demonstrated. The IABG structure relaxes the restriction on the band gap bandwidth with respect to the film thickness of previous ABGs demonstration, and permits the introduction of metal layers, which are essential in routing electrical signal to MEMS devices. Furthermore, the direct integration of electro-acoustic transducers in the same plane of the IABGs facilitates the demonstration of PC-based devices.

This first IABG prototype structure can be extended to many fields of application. For instance, the presence of metal electrodes permits the direct inclusion of IABGs as anchors in piezoelectric contour-mode resonators. Instead of suspending CMRs with narrow beams, the IABG can provide a more mechanically robust anchor that will not alter the device $Q$ by prohibiting the escape of any acoustic wave from the resonator. In RF communications, high $Q$, wideband and miniaturized components can be synthesized out of IABGs to perform frequency multiplexing and spectrum analysis.

Future work on the IABG structures aims at improving the fabrication tolerances to scale the IABG operation into the ultra high frequency $(>1 \mathrm{GHz})$ range, and realizing $\mathrm{RF}$ signal processors by demonstrating components such as filters, waveguides, and multiplexers.

\section{ACKNOWLEDGMENTS}

The authors would like to offer special thanks to the Wolf Nanofabrication Lab staff at Penn for their help with part of the fabrication steps and the Tegal Corporation for the AlN deposition. Also, we greatly appreciate Dr. Watson and Dr. Gaevski for their assistance in platinum deposition at the PRISM Micro/Nanofabrication lab in Princeton.

\section{REFERENCES}

[1] National \& Kapodistrian University of Athens. (2008 Mar.). Phononic Crystals - Database. [Online]. Available: http://www.phys.uoa.gr/phononics/PhononicDatabase1.ht $\mathrm{ml}$

[2] Y. Pennec, et al., "Acoustic channel drop tunneling in a phononic crystal", Appl. Phys, Lett., vol. 87, 261912, 2005 [3] R. Olsson III, I. El-Kady, "Microfabricated phononic crystal devices and applications", Meas. Sci. Technol., vol. 20, 012002, 2009

[4] S. Mohammadi, et al., "Evidence of large high frequency complete phononic band gaps in silicon phononic crystal plates", Appl. Phys. Lett., vol. 92, 221905, 2008

[5] S. Mohammadi, et al., "Complete phononic bandgaps and bandgap maps in two-dimensional silicon phononic crystal plates", Elect. Lett., vol. 43, issue 16, pp. 898-899, 2007

[6] T. Gorishnyy, et al., "Hypersonic Phononic Crystals", Phys. Rev. Lett., vol. 94, 115501, 2005

[7] P. Langlet, et al., "Analysis of the propagation of plane acoustic waves in passive periodic materials using the finite element method", J. Acoust. Soc. Am., vol. 98, issue 5, pp. 2792-2800, 1995

[8] C. Kittel, Introduction to Solid State Physics, John Wiley \& Sons, Inc., New Jersey, 2005

[9] C. Zuo, et al., "Multi-Frequency Pierce Oscillators Based On Piezoelectric AlN Contour-Mode MEMS Resonators", Frequency Control Symposium, 2008 International, May 19-21, 2008, pp. 402-407.

[10] J. Kuypers, et al., "Intrinsic Temperature Compensation of Aluminum Nitride Lamb Wave Resonators for Mulit-Frequency References", Frequency Control Symposium, 2008 International, May 19-21, 2008, pp. 240-249.

\section{CONTACT}

* N. Kuo, tel: +1-215-573-3276; kuo1@ seas.upenn.edu 